\title{
Collapsing Partial Combinatory Algebras
}

\author{
Inge Bethke ${ }^{1,2}$ and Jan Willem Klop ${ }^{1,3}$ \\ 1 CWI, P.O. Box 94079, 1090 GB Amsterdam, The Netherlands \\ 2 University of Utrecht, Department of Philosophy, P.O. Box 80126, \\ 3508 TC Utrecht, The Netherlands \\ 3 Vrije Universiteit, Department of Mathematics and Computer Science, \\ de Boelelaan 1081a, 1081 HV Amsterdam, The Netherlands
}

\begin{abstract}
Partial combinatory algebras occur regularly in the literature as a framework for an abstract formulation of computation theory or recursion theory. In this paper we develop some general theory concerning homomorphic images (or collapses) of pca's, obtained by identification of elements in a pca. We establish several facts concerning final collapses (maximal identification of elements). 'En passant' we find another example of a pca that cannot be extended to a total one.
\end{abstract}

\section{Introduction}

A partial combinatory algebra (pca) is a structure $\mathfrak{A}=\langle A, s, k, \cdot\rangle$ where $A$ is a set, - is a partial binary operation (application) on $A$, and $k, s$ are two elements of $A$ such that

1. $\forall a, a^{\prime} \in A(k \cdot a) \cdot a^{\prime}=a$,

2. $\forall a, a^{\prime} \in A \quad(s \cdot a) \cdot a^{\prime} \downarrow$

3. $\forall a, a^{\prime}, a^{\prime \prime} \in A \quad\left((s \cdot a) \cdot a^{\prime}\right) \cdot a^{\prime \prime}=\left\{\begin{array}{l}\left(a \cdot a^{\prime \prime}\right) \cdot\left(a^{\prime} \cdot a^{\prime \prime}\right) \text { if }\left(a \cdot a^{\prime \prime}\right) \cdot\left(a^{\prime} \cdot a^{\prime \prime}\right) \downarrow \\ \text { undefined }\end{array}\right.$

4. $k \neq s$.

Here $M \downarrow$ means the expression $M$ is defined, and $M=N$ means both expressions are defined and equal. Another useful notation is to write $M \uparrow$ if $M$ is undefined. It is common to omit · and associate unparenthesized expressions to the left. In working with expressions that may or may not be defined, it is useful to write $M \simeq N$ to mean that if either $M$ or $N$ is defined, then both are defined and equal. These notational conventions allow us to replace clause 3 by

$$
\forall a, a^{\prime}, a^{\prime \prime} \in A \quad s a a^{\prime} a^{\prime \prime} \simeq a a^{\prime \prime}\left(a^{\prime} a^{\prime \prime}\right) .
$$

Total pca's ( $c a$ 's), where application is a total operation on the carrier set, are extensively studied in the context of models of $\lambda$-calculus and Combinatory Logic (CL) (cf. e.g. [Bar84], [HS86]); nontotal pca's (nca's), where application is not defined everywhere, are a little less well-off in this respect. They figure in the semantics of programming languages (see the forthcoming book by Mitchell [Mit9?]) as well as in the formalization of constructive mathematics (see [Bee85], [TvD88]). In fact, they are the models of a 'minimal axiomatic basis for theories 
of operators', as stated in [TvD88]. An early approach to treat abstract computation theory was given by the notion of Wagner [Wag69] and Strong [Str68], URS (Uniform Reflexive Structure). More recently, the notion of Effective Applicative Structure, EAS, has been introduced by Asperti and Ciabattoni [AC95]); they show that this notion is in fact equivalent to PCA.

Let us briefly indicate why a study of pca's falls in the scope of higher-order algebra, logic and term rewriting - the subject of the present conference. The connection with term rewriting, via $C L$ and $\lambda$-calculus, is obvious since pca's admit abstraction $[x] M$; in fact they were 'designed' just for that purpose. The connection with higher-order algebra is less clear, also due to the fact that there is no sharp definition of this notion. Meinke [Mei95] bases his survey of higherorder algebra on type theories. Indeed, it is shown that the finite type hierarchy HEO can be built over an arbitrary pca (Bethke [Bet91]); also Mitchell [Mit9?] generalizes the construction of HRO to arbitrary pca's. Furthermore, pca's play a role in the construction of per models for realizability. See also Streicher [Str91].

While nca's thus have enjoyed quite some attention as a tool in abstract computation theory, amazingly little is known about their structural properties. Thus, it was even an open question in [Swan79] whether an nca can always be extended to a ca. A negative answer is given in [Klo82] and [Bet87]. Dually to extending pca's, one may ask what behaviour pca's exhibit under homomorphic images. To be more precise, given a pca $\mathfrak{A}=\langle A, s, k, \cdot\rangle$ and some elements $a, a^{\prime}$ of $A$, one may ask whether there exists a homomorphic image $\phi(\mathfrak{A})$ such that $\phi(a)=\phi\left(a^{\prime}\right)$.

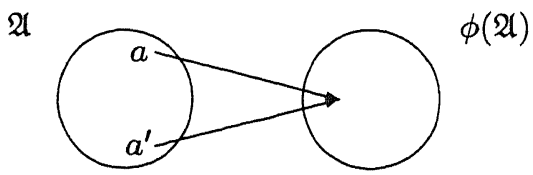

We shall call such a homomorphic image a collapse. There exist several investigations into collapses of ca's (cf. e.g. [Jac75], [JZ85], [BI93]). Here the leading question is whether, given $\lambda$-terms $M$ and $N$, the equation $M=N$ can be added consistently to the $\lambda$-calculus. Considerations of collapses of nca's seem to be rare. In fact, we do not know of any. In the present note, we address this last theme.

Instead of considering collapses, one can also study certain well-behaved congruence relations. As it turns out, there exists a natural 1-1 correspondence between these relations and collapses: every such congruence induces a collapse and vice versa. We establish this fact in Sect. 2.

We use the correspondence between well-behaved congruence relations and collapses in Sect. 3 to show that there is at least one major difference between nca's and ca's with respect to their class of collapses: nca's always have a final collapse $\phi_{\text {fin }}(\mathfrak{A})$ which combines all possible identifications. 


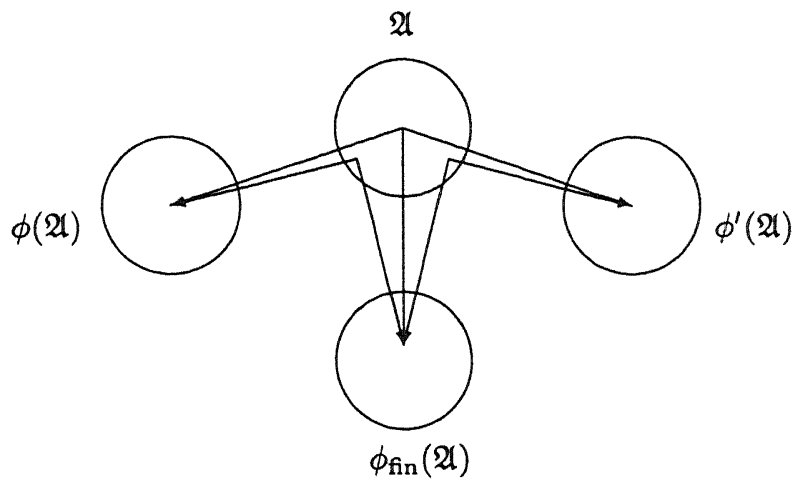

For ca's, such a final collapse does not need to exist. We provide a counterexample.

Not every pca allows for additional identifications. In Sect. 4, we give two examples of these irreducible pca's: the well-known total graph models as well as the nontotal pca of natural numbers with partial recursive function application cannot be collapsed any further.

In Sect. 5, we concentrate on extensional collapses, i.e. collapses that identify elements displaying identical applicative behaviour. We provide a condition on nca's that guarantees the existence of extensional collapses. In fact, if an nca meets this condition, then its final collapse is extensional. As an application which may be of independent interest, we show that the paradigmatic nca of strongly normalizing CL-terms has an extensional final collapse.

\section{Collapses of PCA's}

A homomorphism is a structure-preserving map from one algebra to another. For partial algebras, there is one basic notion for a homomorphism which simultaneously generalizes the notions of homomorphisms between total algebras and relational structures respectively. However, since its defining property is relatively weak, we select a proper subclass of homomorphisms throughout this paper. An extensive survey of the model theory of partial algebras can be found in [Bur82].

Definition 1. Let $\mathfrak{A}=\langle A, s, k, \cdot\rangle$ and $\mathfrak{B}=\left\langle B, s^{\prime}, k^{\prime}, \cdot^{\prime}\right\rangle$ be pca's.

1. A closed homomorphism of $\mathfrak{A}=\langle A, s, k, \cdot\rangle$ into $\mathfrak{B}=\left\langle B, s^{\prime}, k^{\prime}, \cdot^{\prime}\right\rangle$ is a mapping $\phi: A \rightarrow B$ such that

(a) $\phi(s)=s^{\prime}, \phi(k)=k^{\prime}$, and

(b) $\phi\left(a \cdot a^{\prime}\right) \simeq \phi(a) \cdot^{\prime} \phi\left(a^{\prime}\right)$ for all $a, a^{\prime} \in A$.

If $\phi$ is surjective, then $\phi$ is a closed epimorphism, and if $\phi$ is bijective, then $\phi$ is an isomorphism.

2. $\phi$ is a collapse of $\mathfrak{A}$ if $\phi$ is a closed epimorphism of $\mathfrak{A}$ onto some pca $\mathfrak{B}$. 
We write $\operatorname{Col}(\mathfrak{A})$ for the class of collapses of $\mathfrak{A}$.

A trivial example of a collapse is the identity map from $A$ to $A$. Instead of considering collapses of $\mathfrak{A}$, one can also study congruence relations on $A$, i.e. equivalence relations with the added property that application relates related elements.

Definition 2. Let $\mathfrak{A}=\langle A, s, k, \cdot\rangle$ be a pca. The set of contexts over $\mathfrak{A}, \mathcal{C}_{\mathfrak{A}}$, is defined as follows.

1. $\square \in \mathcal{C}_{\mathfrak{A}}$, and

2. if $C \in \mathcal{C}_{\mathfrak{A}}$ and $a \in A$, then $a C \in \mathcal{C}_{\mathfrak{A}}$ and $C a \in \mathcal{C}_{\mathfrak{A}}$.

If $C$ is a context, then $C[a]$ denotes the expression obtained from $C$ by replacing $\square$ by $a$.

Definition 3. Let $\mathfrak{A}=\langle A, s, k, \cdot\rangle$ be a pca and $E$ be an equivalence relation on A.

1. $E$ is called proper if $\langle s, k\rangle \notin E$.

2. $E$ is said to be a congruence if for all $\left\langle a, a^{\prime}\right\rangle \in E$ and $C \in \mathcal{C}_{\mathfrak{A}}$, if either $C[a]$ or $C\left[a^{\prime}\right]$ is defined, then both are defined and $\left\langle C[a], C\left[a^{\prime}\right]\right\rangle \in E$.

We write $\operatorname{Con}(\mathfrak{A})$ for the set of proper congruence relations on $A$.

A trivial example of a proper congruence on $A$ is the diagonal $\{\langle a, a\rangle \mid a \in$ $A\}$. However, there may be more complex ones. In particular, every collapse corresponds to a congruence relation, namely the one that relates identified elements.

Definition 4. Let $\mathfrak{A}=\langle A, s, k, \cdot\rangle$ be a pca and $\phi \in \operatorname{Col}(\mathfrak{A})$. Put

$$
E_{\phi}=\left\{\left\langle a, a^{\prime}\right\rangle \in A \times A \mid \phi(a)=\phi\left(a^{\prime}\right)\right\} .
$$

Proposition 5. Let $\mathfrak{A}=\langle A, s, k, \cdot\rangle$ be a pca and $\phi \in \operatorname{Col}(\mathfrak{A})$. Then $E_{\phi} \in$ $\operatorname{Con}(\mathfrak{A})$.

Proof. $E_{\phi}$ is clearly an equivalence relation on $A$ and is proper, since $\phi(s)=$ $s^{\prime} \neq k^{\prime}=\phi(k)$. To prove that $E_{\phi}$ is a congruence, let $\left\langle a, a^{\prime}\right\rangle \in E_{\phi}$ and $C \in \mathcal{C}_{\mathfrak{a}}$. Then

$$
\phi(C[a]) \simeq C^{\prime}[\phi(a)] \simeq C^{\prime}\left[\phi\left(a^{\prime}\right)\right] \simeq \phi\left(C\left[a^{\prime}\right]\right)
$$

for some context $C^{\prime}$. Hence $C[a]$ is defined if and only if $C\left[a^{\prime}\right]$ is defined, and if they are both defined, then $\left\langle C[a], C\left[a^{\prime}\right]\right\rangle \in E_{\phi}$.

Given any congruence relation $E$ on $A$, we may construct a pca $\mathfrak{A} / E$ of $\mathfrak{A}$ called the quotient of $\mathfrak{A}$ modulo $E$. The intuitive idea behind $\mathfrak{A} / E$ is that we identify related elements of $A$. 
Definition 6. Let $\mathfrak{A}=\langle A, s, k, \cdot\rangle$ be a pca and $E \in \operatorname{Con}(\mathfrak{A})$. We form the quotient

$$
\mathfrak{A} / E=\left\langle A / E,[s]_{E},[k]_{E}, \cdot E\right\rangle
$$

by taking the collection $A / E=\left\{[a]_{E} \mid a \in A\right\}$ of equivalence classes $[a]_{E}=\left\{a^{\prime} \mid\right.$ $\left.\left\langle a, a^{\prime}\right\rangle \in E\right\}$ equipped with the application operation

$$
[a]_{E} \cdot E\left[a^{\prime}\right]_{E}=\left\{\begin{array}{ll}
{\left[a a^{\prime}\right]_{E}} & \text { if } a a^{\prime} \downarrow \\
\text { undefined otherwise }
\end{array} .\right.
$$

Proposition 7. Let $\mathfrak{A}=\langle A, s, k, \cdot\rangle$ be a pca. For $E \in \operatorname{Con}(\mathfrak{A}), \lambda a \in A \cdot[a]_{E} \in$ $\operatorname{Col}(\mathfrak{A})$.

Proof. We first show that $\cdot_{E}$ is well-defined. To this end, let $[a]_{E}=\left[a^{\prime}\right]_{E}$ and $[b]_{E}=\left[b^{\prime}\right]_{E}$. Then $\left\langle a, a^{\prime}\right\rangle,\left\langle b, b^{\prime}\right\rangle \in E$. Let $C \equiv \square b, C^{\prime} \equiv a^{\prime} \square$. As $E$ is a congruence, it follows that $C[a] \downarrow$ iff $C\left[a^{\prime}\right] \downarrow$, and $C^{\prime}[b] \downarrow$ iff $C^{\prime}\left[b^{\prime}\right] \downarrow$. Thus

$$
a b \downarrow \leftrightarrow a^{\prime} b \downarrow \leftrightarrow a^{\prime} b^{\prime} \downarrow
$$

Hence $[a]_{E}[b]_{E} \downarrow$ iff $\left[a^{\prime}\right]_{E}\left[b^{\prime}\right]_{E} \downarrow$. Now assume $[a]_{E}[b]_{E} \downarrow$. Then $\left\langle a b, a^{\prime} b\right\rangle,\left\langle a^{\prime} b, a^{\prime} b^{\prime}\right\rangle \in$ $E$. So $\left\langle a b, a^{\prime} b^{\prime}\right\rangle \in E$, i.e. $[a b]_{E}=\left[a^{\prime} b^{\prime}\right]_{E}$. Thus $[a]_{E}[b]_{E}=\left[a^{\prime}\right]_{E}\left[b^{\prime}\right]_{E}$.

$\mathfrak{A} / E$ meets the first three conditions on pca's, since $\mathfrak{A}$ is a pca; it meets the last condition, since $E$ is proper. Hence $\mathfrak{A} / E$ is a pca. Clearly, $\lambda a \in A$. $[a]_{E}$ is a closed epimorphism of $\mathfrak{A}$ onto $\mathfrak{A} / E$.

If we, as is standard, identify isomorphic pca's, we can in fact pass in this way from collapses to proper congruence relations and back, and end up were we have started. This is a special case of the well-known First Homomorphism Theorem of universal algebra (see e.g. [Grä79]). Thus, given collapses $\phi, \phi^{\prime}$ of $\mathfrak{A}$, let us write $\phi \cong \phi^{\prime}$ if the homomorphic images of $\mathfrak{A}$ under $\phi$ and $\phi^{\prime}$ are isomorphic.

Theorem 8. Let $\mathfrak{A}=\langle A, s, k, \cdot\rangle$ be a pca. Then

1. $\lambda a \in A \cdot[a]_{E_{\phi}} \cong \phi$ for all $\phi \in \operatorname{Col}(\mathfrak{A})$, and

2. $E_{\lambda a \in A .[a]_{E}}=E$ for all $E \in \operatorname{Con}(\mathfrak{A})$.

Proof. To prove 1., define the surjection $\psi: A / E_{\phi} \rightarrow \phi(A)$ by $\psi\left([a]_{E_{\phi}}\right)=\phi(a)$. As

$$
[a]_{E_{\phi}}=\left[a^{\prime}\right]_{E_{\phi}} \leftrightarrow\left\langle a, a^{\prime}\right\rangle \in E_{\phi} \leftrightarrow \phi(a)=\phi\left(a^{\prime}\right),
$$

it follows that $\psi$ is well-defined and bijective, and since $\phi$ is a closed homomorphism, $\psi$ is a closed homomorphism too. So $\psi$ is an isomorphism. For 2., note that

$$
E_{\lambda a \in A .[a]_{E}}=\left\{\left\langle a, a^{\prime}\right\rangle \in A \times A \mid[a]_{E}=\left[a^{\prime}\right]_{E}\right\}=E
$$




\section{Final Collapses of PCA's}

A pca $\mathfrak{A}$ has always an initial collapse, i.e. a collapse $\phi$ such that for any collapse $\phi^{\prime}$ there is a unique homomorphism $\psi$ with $\psi \circ \phi=\phi^{\prime}$. This initial collapse is just the identity on $A$ that does not identify any elements. Nca's, however, also have a final collapse, i.e. a collapse $\phi$ such that for any collapse $\phi^{\prime}$ there is a unique homomorphism $\psi$ with $\psi \circ \phi^{\prime}=\phi$. Such a final collapse then identifies all elements that can be identified. The crucial observation is the following.

Proposition 9. Let $\mathfrak{A}=\langle A, s, k, \cdot\rangle$ be an nca and $E$ be an equivalence relation on $A$. Then $E$ is proper provided $E$ is a congruence relation.

Proof. Assume $E$ is a congruence and suppose that $\langle s, k\rangle \in E$. Pick $a, a^{\prime} \in A$ such that $a a^{\prime} \uparrow$ and let $C \equiv \square k a a^{\prime}$. Then $s k a a^{\prime} \downarrow$ iff $k k a a^{\prime} \downarrow$. As $k k a a^{\prime}=k a^{\prime}$, it follows that $s k a a^{\prime}=k a^{\prime}\left(a a^{\prime}\right)$. Hence $a a^{\prime} \downarrow$. Contradiction.

In dealing with nca's, we can therefore forget about properness and concentrate on congruence only. As it turns out, the union of all congruences is again a congruence.

Definition 10. Let $\mathfrak{A}=\langle A, s, k, \cdot\rangle$ be an nca. Put

$$
E_{\text {fin }}=\left\{\left\langle a, a^{\prime}\right\rangle \in A \times A \mid \forall C \in \mathcal{C}_{\mathfrak{A}} C[a] \downarrow \text { if and only if } C\left[a^{\prime}\right] \downarrow\right\} .
$$

Lemma 11. Let $\mathfrak{A}=\langle A, s, k, \cdot\rangle$ be an nca. Then

1. $E_{\text {fin }} \in \operatorname{Con}(\mathfrak{A})$,

2. $E_{\mathrm{fin}}=\bigcup \operatorname{Con}(\mathfrak{A})$.

Proof. 1. $E_{\mathrm{fin}}$ is clearly a congruence relation. Hence $E_{\mathrm{fin}} \in \mathrm{Con}(\mathfrak{A})$ by Proposition 9.

2. From 1. it follows that $E_{\text {fin }} \subseteq \bigcup \operatorname{Con}(\mathfrak{A})$. For the other inclusion, let $\left\langle a, a^{\prime}\right\rangle \in$ $\cup \operatorname{Con}(\mathfrak{A})$. Then $\left\langle a, a^{\prime}\right\rangle \in E$ for some $E \in \operatorname{Con}(\mathfrak{A})$. Thus, since $E$ is a congruence, $\left\langle a, a^{\prime}\right\rangle \in E_{\mathrm{fin}}$.

Theorem 12. Every nca $\mathfrak{A}=\langle A, s, k, \cdot\rangle$ has a final collapse.

Proof. We shall prove that $\lambda a \in A \cdot[a]_{E_{\text {fin }}}$ is final. To this end, let $\phi$ be any collapse of $\mathfrak{A}$ onto some pca $\mathfrak{B}=\left\langle B, s^{\prime}, k^{\prime}, .^{\prime}\right\rangle$ and put $\psi(b)=[a]_{E_{\text {fin }}}$ where $\phi(a)=b$. Observe that $\psi$ is well-defined. For, if $\phi(a)=b=\phi\left(a^{\prime}\right)$, then $\left\langle a, a^{\prime}\right\rangle \in$ $E_{\phi} \subseteq E_{\text {fin }}$ and hence $[a]_{E_{\text {fin }}}=\left[a^{\prime}\right]_{E_{\text {fin }}}$. Clearly $\psi$ is a homomorphism. And as $\psi(\phi(a))=[a]_{E_{\mathrm{fin}}}$ for all $a \in A$, it follows that $\psi \circ \phi=\lambda a \in A .[a]_{E_{\mathrm{fin}}}$. Now let $\psi^{\prime}$ be such that $\psi^{\prime} \circ \phi=\lambda a \in A .[a]_{E_{\mathrm{fin}}}$. Then $\psi(\phi(a))=\psi^{\prime}(\phi(a))$ for all $a \in A$. Hence $\psi(b)=\psi^{\prime}(b)$ for all $b \in B$. So $\psi=\psi^{\prime}$.

For ca's, such a final collapse does not need to exist. To see this, we recall a well-known result from [Jac75]. Extensional combinatory logic, ECL, is an equational theory consisting of expressions of the form $M=N$ where $M$ and $N$ 
are terms constructed as usual from variables, the two constants $S$ and $K$, and a binary application operator - which we do not write. The axioms and rules of inference of ECL are those of equational logic together with the axioms

$$
K x y=x \quad S x y z=x z(y z)
$$

and the rule

$$
\frac{M x=N x}{M=N}
$$

where the variable $x$ occurs in neither $M$ nor $N$. Closed terms modulo provable equality form a ca in the following way: We let

$$
\mathfrak{A}_{\mathrm{ECL}}=\left\langle T^{0} / \mathrm{ECL},[S]_{\mathrm{ECL}},[K]_{\mathrm{ECL}}, \cdot\right\rangle
$$

where $T^{0}$ is the set of closed terms (i.e. the set of terms without any variable),

$$
\begin{gathered}
T^{0} / \mathrm{ECL}=\left\{[M]_{\mathrm{ECL}} \mid M \in T^{0}\right\} \\
{[M]_{\mathrm{ECL}}=\left\{N \in T^{0} \mid \mathrm{ECL} \vdash M=N\right\}}
\end{gathered}
$$

and

$$
[M]_{\mathrm{ECL}} \cdot[N]_{\mathrm{ECL}}=[M N]_{\mathrm{ECL}} .
$$

In [Jac75], Jacopini - using a slightly different terminology - proved that $[\Omega]_{\mathrm{ECL}}$, where

$$
\Omega \equiv S(S K K)(S K K)(S(S K K)(S K K))
$$

can be identified with any other element in this ca. This means in particular that $\mathfrak{A}_{\mathrm{ECL}}$ has collapses $\phi$ and $\phi^{\prime}$ such that $\phi\left([\Omega]_{\mathrm{ECL}}\right)=\phi\left([S]_{\mathrm{ECL}}\right)$ and $\phi^{\prime}\left([\Omega]_{\mathrm{ECL}}\right)=$ $\phi^{\prime}\left([K]_{\mathrm{ECL}}\right)$. It follows that $\mathfrak{A}_{\mathrm{ECL}}$ lacks a final collapse. For, suppose $\mathfrak{A}_{\mathrm{ECL}}$ has a final collapse onto some pca $\mathfrak{B}=\left\langle B, s^{\prime}, k^{\prime}, \cdot^{\prime}\right\rangle$. Then there are homomorphisms $\psi$ and $\psi^{\prime}$ such that $\psi \circ \phi=\psi^{\prime} \circ \phi^{\prime}$. So

$s^{\prime}=\psi\left(\phi\left([S]_{\mathrm{ECL}}\right)\right)=\psi\left(\phi\left([\Omega]_{\mathrm{ECL}}\right)\right)=\Omega^{\prime}=\psi^{\prime}\left(\phi^{\prime}\left([\Omega]_{\mathrm{ECL}}\right)\right)=\psi^{\prime}\left(\phi^{\prime}\left([K]_{\mathrm{ECL}}\right)\right)=k^{\prime}$

where $\Omega^{\prime} \equiv s^{\prime}\left(s^{\prime} k^{\prime} k^{\prime}\right)\left(s^{\prime} k^{\prime} k^{\prime}\right)\left(s^{\prime}\left(s^{\prime} k^{\prime} k^{\prime}\right)\left(s^{\prime} k^{\prime} k^{\prime}\right)\right)$. This constitutes a contradiction with the fact that the homomorphic image of $\mathfrak{A}_{\mathrm{ECL}}$ under the final collapse meets the last condition on pca's.

Theorem 13. Not every ca has a final collapse. 


\section{Irreducible PCA's}

Not every pca allows for further identifications. For example, the codomain of every final collapse has reached its maximal degree of identifications. We shall call such a pca, where the only collapse is the trivial initial one, irreducible.

Definition 14. Let $\mathfrak{A}=\langle A, s, k, \cdot\rangle$ be a pca. $\mathfrak{A}$ is irreducible if $E_{\phi} \subseteq\{\langle a, a\rangle \mid$ $a \in A\}$ for every collapse $\phi$ of $\mathfrak{A}$.

There are prominent pca's which share this property. We give two examples.

Example 1. The first example uses only elementary properties of sets, and is directly taken from Engeler [Eng81]. It is in fact a notational variant of one of several ca's first described in Plotkin [Plo72] which in turn are nearly the same as the better known $P \omega$ construction of Scott [Sco76].

Let $A$ be any nonempty set, and let $B$ be the least set containing $A$ and all ordered pairs consisting of a finite subset $\beta \subseteq B$ and an element $b \in B$. Assume that elements of $A$ are distinguishable from ordered pairs. Let $D_{A}$ be the power set of $B$, and define the total application operation on $D_{A}$ by

$$
x y=\{b \in B \mid(\beta, b) \in x \text { for some } \beta \subseteq y\} .
$$

Choose

$$
s=\{(\alpha,(\beta,(\gamma, b))) \mid b \in \alpha \gamma(\beta \gamma)\}
$$

and

$$
k=\{(\alpha,(\beta, b)) \mid b \in \alpha\} .
$$

Then $\mathfrak{D}=\left\langle D_{A}, s, k, \cdot\right\rangle$ is a ca.

To prove that $\mathfrak{D}$ is irreducible, let $E_{\phi}$ be any collapse of $\mathfrak{D}$ and let $\langle x, y\rangle \in E_{\phi}$. Assume $x \neq y$. Say, $b \in x$ and $b \notin y$ for some $b \in B$. Define

$$
z=\left\{\left(\{b\}, b^{\prime}\right) \mid b^{\prime} \in k\right\} \text {. }
$$

Then $z \in D_{A}$. Now let $C$ be the context $z \square$. Since $E_{\phi}$ is a congruence, $\langle z x, z y\rangle \in$ $E_{\phi}$. Observe that $z x=k$ and $z y=\emptyset$. Hence $\langle k, \emptyset\rangle \in E_{\phi}$, and therefore $\langle k s s, \emptyset s s\rangle \in E_{\phi}$. That is, also $\langle s, \emptyset\rangle \in E_{\phi}$. It follows that $\langle s, k\rangle \in E_{\phi}$. Thus $E_{\phi}$ is improper. This is a contradiction. So $x=y$; whence $\langle x, y\rangle \in\left\{\langle x, x\rangle \mid x \in D_{A}\right\}$.

Remark. The argument given above extends to the family of $P \omega$-models which consists of coded versions of $\mathfrak{D}$. At first sight, this may seem to contradict the remarkable result of Baeten and Boerboom in their 1979 paper $\Omega$ can be anything it shouldn't be (cf. [BB79]). The authors, however, do not consider collapses. Rather they show that, given an arbitrary closed $\lambda$-term $M$, there exists a member of the $P \omega$-family which identifies $M$ and $\Omega$. 
Example 2. As second example we consider the nca of natural numbers with partial recursive function application. More specifically, we define a nontotal application operation on the natural numbers $\mathbb{N}$ by

$$
n m=\{n\}(m)
$$

where $\{n\}$ is the partial recursive function with Gödel number $n$. We may let $k$ be any Gödel number of the recursive function which, given some argument $x$, returns a Gödel number of the constant function returning $x$. The natural number $s$ is slightly more complicated: we let $s$ be a Gödel number of the recursive function

$$
f(x)=n_{x}
$$

where $n_{x}$ is a Gödel number of the recursive function

$$
g(y)=m_{x, y}
$$

with $m_{x, y}$ a Gödel number of the partial recursive function

$$
h(z) \simeq\{\{x\}(z)\}(\{y\}(z)) .
$$

The existence of this function is easiest to explain using Turing machines, or some other model of computation. Then $\mathfrak{N}=\langle\mathbb{N}, s, k, \cdot\rangle$ is an nca.

To prove that $\mathfrak{N}$ is irreducible, let $E_{\phi}$ be any collapse of $\mathfrak{N}$ and let $\langle x, y\rangle \in E_{\phi}$. Assume $x \neq y$. It is now not hard to imagine a partial recursive function $g$ with Gödel number $z$, say, such that $g(x) \uparrow$ and $g(y) \downarrow$. Then $C \equiv z \square$ is a context with $C[x] \uparrow$ and $C[y] \downarrow$. Thus $E_{\phi}$ is not a congruence. This is a contradiction. So $x=y$; whence $\langle x, y\rangle \in\{\langle x, x\rangle \mid x \in \mathbb{N}\}$.

\section{Extensional Collapses of PCA's}

In this final section, we shall consider collapses that identify elements which display identical applicative behaviour.

Definition 15. Let $\mathfrak{A}=\langle A, s, k, \cdot\rangle$ be a pca.

1. $\mathfrak{A}$ is extensional if for all $a, a^{\prime} \in A$,

$$
\left(\forall a^{\prime \prime} \in A a a^{\prime \prime} \simeq a^{\prime} a^{\prime \prime}\right) \rightarrow a=a^{\prime} .
$$

2. $\mathfrak{A}$ has an extensional collapse if $\mathfrak{A}$ has a collapse onto some extensional pca.

Proposition 16. Let $\mathfrak{A}=\langle A, s, k, \cdot\rangle$ be a pca and let $\phi$ be an extensional collapse of $\mathfrak{A}$. Then

$$
\left\{\left\langle a, a^{\prime}\right\rangle \in A \times A \mid \forall a^{\prime \prime} \in A a a^{\prime \prime} \simeq a^{\prime} a^{\prime \prime}\right\} \subseteq E_{\phi} .
$$


Proof. Suppose $\phi$ is a collapse onto the extensional pca $\mathfrak{B}=\left\langle B, s^{\prime}, k^{\prime}, .^{\prime}\right\rangle$. Let $a, a^{\prime} \in A$ be such that $a a^{\prime \prime} \simeq a^{\prime} a^{\prime \prime}$ for all $a^{\prime \prime} \in A$ and let $b \in B$. Say, $b=\phi\left(a^{\prime \prime}\right)$. Then

$$
\phi(a) b \simeq \phi(a) \phi\left(a^{\prime \prime}\right) \simeq \phi\left(a a^{\prime \prime}\right) \simeq \phi\left(a^{\prime} a^{\prime \prime}\right) \simeq \phi\left(a^{\prime}\right) \phi\left(a^{\prime \prime}\right) \simeq \phi\left(a^{\prime}\right) b .
$$

Hence $\phi(a)=\phi\left(a^{\prime}\right)$, since $\mathfrak{B}$ is extensional. Therefore $\left\langle a, a^{\prime}\right\rangle \in E_{\phi}$.

Not every pca has an extensional collapse. Observe, for example, that the two pca's considered in Example 1 and 2 are not extensional. As they are both irreducible, it follows that they do not have an extensional collapse.

Theorem 17. Not every pca has an extensional collapse.

For nca's, there exists a simple condition such that the final collapse is extensional.

Theorem 18. Let $\mathfrak{A}=\langle A, s, k, \cdot\rangle$ be an nca. Its final collapse is extensional if and only if

(†) $\forall a, a^{\prime} \in A\left(\forall C \in \mathcal{C}_{\mathfrak{a}} \forall a^{\prime \prime} \in A\left(C\left[a a^{\prime \prime}\right] \downarrow \leftrightarrow C\left[a^{\prime} a^{\prime \prime}\right] \downarrow\right) \rightarrow\left\langle a, a^{\prime}\right\rangle \in E_{\mathrm{fin}}\right)$.

Proof. Suppose ( $\dagger$ ) holds. To prove that $\mathfrak{A} / E_{\text {fin }}$ is extensional, let $[a]_{E_{\text {fin }}},\left[a^{\prime}\right]_{E_{\text {fin }}} \in$ $A / E_{\mathrm{fin}}$ be such that

$$
[a]_{E_{\mathrm{fin}}}\left[a^{\prime \prime}\right]_{E_{\mathrm{fin}}} \simeq\left[a^{\prime}\right]_{E_{\mathrm{fin}}}\left[a^{\prime \prime}\right]_{E_{\mathrm{fin}}}
$$

for every $\left[a^{\prime \prime}\right]_{E_{\mathrm{fin}}} \in A / E_{\mathrm{fin}}$. Now let $a^{\prime \prime} \in A, C$ be any context and assume one of $C\left[a a^{\prime \prime}\right]$ and $C\left[a^{\prime} a^{\prime \prime}\right]$ is defined, say $C\left[a a^{\prime \prime}\right] \downarrow$. Then $a a^{\prime \prime} \downarrow$ and hence $\left[a a^{\prime \prime}\right]_{E_{\text {fin }}}=$ $\left[a^{\prime} a^{\prime \prime}\right]_{E_{\mathrm{fin}}}$. So $\left\langle a a^{\prime \prime}, a^{\prime} a^{\prime \prime}\right\rangle \in E_{\mathrm{fin}}$ and therefore $C\left[a^{\prime} a^{\prime \prime}\right] \downarrow$. Thus $\left\langle a, a^{\prime}\right\rangle \in E_{\mathrm{fin}}$ by $(\dagger)$, i.e.

$$
[a]_{E_{\text {fin }}}=\left[a^{\prime}\right]_{E_{\text {fin }}} .
$$

For the other direction, assume $\mathfrak{A} / E_{\mathrm{fin}}$ is extensional and let $a, a^{\prime} \in A$ be such that $C\left[a a^{\prime \prime}\right] \downarrow$ if and only if $C\left[a^{\prime} a^{\prime \prime}\right] \downarrow$ for all contexts $C$ and all $a^{\prime \prime} \in A$. Then, in particular,

$$
[a]_{E_{\text {fin }}}\left[a^{\prime \prime}\right]_{E_{\text {fin }}} \simeq\left[a^{\prime}\right]_{E_{\text {fin }}}\left[a^{\prime \prime}\right]_{E_{\text {fin }}}
$$

for every $\left[a^{\prime \prime}\right]_{E_{\mathrm{fin}}} \in A / E_{\mathrm{fin}}$. Hence $[a]_{E_{\mathrm{fin}}}=\left[a^{\prime}\right]_{E_{\mathrm{fin}}}$, since $\mathfrak{A} / E_{\mathrm{fin}}$ is extensional. So $\left\langle a, a^{\prime}\right\rangle \in E_{\text {fin }}$.

We shall apply this result in the next and final example of this paper where we prove that the final collapse of the nca of closed, strongly normalizing CL-terms is extensional. In the example, we employ fundamental definitions and notions of term rewrite systems. Extensive surveys of term rewriting can be found in [Klo92] and [DJ90].

Example 3. Reduction in CL is generated by the rules

1. $S L M N \rightarrow L N(M N)$ 
2. $K L M \rightarrow L$

for all CL-terms $L, M, N$. Here 'generated' means:

3. if $L \rightarrow M$ then $C[L] \rightarrow C[M]$

for every context $C$. Contexts are defined as in Definition 2 with element $a$ changed into CL-term $L$.

We write $L \equiv M$ if $L$ and $M$ are identical terms. The transitive-reflexive closure of the rewrite relation $\rightarrow$ is denoted by $\rightarrow$. If $L \rightarrow M$, we say that $L$ reduces to $M$. The equivalence relation generated by $\rightarrow$ is called convertibility and written as $=$.

A term of the form $S L M N$ or $K L M$ is a redex; its contractum is $L N(M N)$ or $L$, respectively. A term not containing such redexes is a normal form ( $n f)$ and has a $n f$ if it reduces to one. A reduction of $L$ is a sequence of terms $L \equiv L_{1} \rightarrow$ $L_{2} \rightarrow L_{3} \rightarrow \cdots$. Reductions may be infinite. If every reduction of $L$ terminates eventually (in a normal form), then $L$ is said to be strongly normalizing. We let $\mathrm{SN}$ be the set of all strongly normalizing CL-terms, and $\mathrm{SN}^{0}$ be the set of all closed, strongly normalizing CL-terms. Observe that $\omega \equiv S(S K K)(S K K) \in$ $\mathrm{SN}^{0}$; however, $\Omega \equiv \omega \omega \notin \mathrm{SN}$.

The rewrite system $\mathrm{CL}$ is orthogonal and has therefore nice properties such as confluence. Another pleasantness is:

(*) Let $L \notin \mathrm{SN}$ and $L \rightarrow M$ be such that $M \in \mathrm{SN}$. Then the redex contracted in the reduction step must contain a proper subterm $N$ with $N \notin \mathrm{SN}$ that is erased in the step $L \rightarrow M$

(cf. Exercise 3.1.13 of [Klo92]). From this we obtain the following proposition.

Proposition 19. Let $C$ be a context and $L, M \in \mathrm{SN}$.

1. If $L \rightarrow M$, then $C[L] \in \mathrm{SN}$ if and only if $C[M] \in \mathrm{SN}$.

2. If $L \rightarrow M$, then $C[L] \in \mathrm{SN}$ if and only if $C[M] \in \mathrm{SN}$.

3. If $L=M$, then $C[L] \in \mathrm{SN}$ if and only if $C[M] \in \mathrm{SN}$.

Proof. 1. If $L \rightarrow M$, then $C[L] \rightarrow C[M]$. Hence $C[M] \in \mathrm{SN}$ if $C[L] \in \mathrm{SN}$. For the other direction, assume $C[M] \in \mathrm{SN}$ and suppose $C[L] \notin \mathrm{SN}$. By $(*)$ there must be a subterm $N$ of $L$ with $N \notin \mathrm{SN}$. This is of course impossible, since $L \in \mathrm{SN}$.

2. Follows from 1 .

3. If $L=M$, then by confluence, $L \rightarrow N \leftarrow M$ for some term $N$. Moreover, $N \in \mathrm{SN}$, since $L, M \in \mathrm{SN}$. Therefore $C[L] \in \mathrm{SN}$ iff $C[N] \in \mathrm{SN}$ iff $C[M] \in \mathrm{SN}$ by 2 .

Closed, strongly normalizing terms modulo convertibility form an nca in the following way: We let

$$
\mathfrak{A}_{\mathrm{SN}}=\left\langle\left\{[M]_{\mathrm{SN}} \mid M \in \mathrm{SN}^{0}\right\},[S]_{\mathrm{SN}},[K]_{\mathrm{SN}}, \cdot\right\rangle
$$


where

$$
[M]_{\mathrm{SN}}=\left\{N \in \mathrm{SN}^{0} \mid M=N\right\}
$$

and

$$
[M]_{\mathrm{SN}} \cdot[N]_{\mathrm{SN}}=\left\{\begin{array}{l}
{[M N]_{\mathrm{SN}} \text { if } M N \in \mathrm{SN},} \\
\text { undefined otherwise }
\end{array}\right.
$$

Observe that application is well-defined. For, if $M=M^{\prime}$ and $N=N^{\prime}$, then $M N \in \mathrm{SN}$ iff $M^{\prime} N \in \mathrm{SN}$ iff $M^{\prime} N^{\prime} \in \mathrm{SN}$ by Proposition 19.3. By a similar argument, $\mathfrak{A}_{\mathrm{SN}}$ satisfies conditions 1 . and 3 . on pca's. Moreover, $S L M \in \mathrm{SN}$ if $L, M \in \mathrm{SN}$. Hence also condition 2. is met. Finally, $[S]_{\mathrm{SN}} \neq[K]_{\mathrm{SN}}$, since $S \not K K$. So $\mathfrak{A}_{\mathrm{SN}}$ is an nca.

To prove that the final collapse of $\mathfrak{A}_{\mathrm{SN}}$ is extensional, we invoke Theorem 18 . That is, we shall prove that for all $L, M \in \mathrm{SN}^{0}$, if

$$
\forall C \in \mathcal{C}_{\mathfrak{A}_{\mathrm{SN}}} \forall N \in \mathrm{SN}^{0}\left(C\left[[L]_{\mathrm{SN}}[N]_{\mathrm{SN}}\right] \downarrow \leftrightarrow C\left[[M]_{\mathrm{SN}}[N]_{\mathrm{SN}}\right] \downarrow\right),
$$

then $\left\langle[L]_{\mathrm{SN}},[M]_{\mathrm{SN}}\right\rangle \in E_{\mathrm{fin}}$. If we denote the set of contexts built from the hole $\square$ and closed, strongly normalizing terms by $\mathcal{C}_{\mathrm{SN}}$, the requirement for Theorem 18 boils down to the following: for all $L, M \in \mathrm{SN}^{0}$, if

$$
\text { (‡) } \forall C \in \mathcal{C}_{\mathrm{SN}} \forall N \in \mathrm{SN}^{0}(C[L N] \in \mathrm{SN} \leftrightarrow C[M N] \in \mathrm{SN}),
$$

then $C[L] \in \mathrm{SN}$ iff $C[M] \in \mathrm{SN}$ for all $C \in \mathcal{C}_{\mathrm{SN}}$. We start with an intuitive description of the proof.

We first recall the notion of descendants of a specific occurrence of a subterm $L$ of $M$ under a reduction $M \rightarrow N$ : we underline the given occurrence of $L$ in $M$ (and nothing else) and perform the reduction $M \rightarrow N$. Then we look for the set of all underlined subterms of $N$. These subterms (actually subterm occurrences) are the descendants of $L$. We moreover say that $L$ is activated in this reduction if $N \equiv C\left[L^{*} P\right]$ for some context $C$ and some term $P$ where $L^{*}$ is a descendant of $L$.

Now suppose $(\ddagger)$ holds and $C[L] \notin \mathrm{SN}$, i.e. $C[L]$ has an infinite reduction. Observe that by Proposition 19.2 we may assume that $L$ is a normal form. This means that the infinite reduction is sustained by just one source: the 'material' present in the context $C$. In the course of the infinite reduction, $L$ will be multiplied in several descendants and the only contribution of $L$ to sustaining the infinite reduction is that a descendant of $L, L^{*}$, is activated such that $L^{*} P$ eventually will develop into a redex and will be contracted.

Indeed, if no descendant of $L$ ever would be activated, all activity would be due to the context. In that case we also have an infinite reduction after replacing $L$ by $M$.

Given the fact that $C[L]$ has an infinite reduction, we want to construct an infinite reduction of $C[M]$. This is done by gradually replacing all descendants of $L$ by $M$, in the following manner: as soon as a descendant of $L$ is activated, we replace it by $M$. Because of $(\ddagger)$, this replacement does not loose the possibility of an infinite reduction. Performing this infinite reduction in the so obtained new context, we again wait until the first of the remaining descendants of $L$ is 
activated and replace it again by $M$. This procedure is repeated ad infinitum. In each step of the procedure, we gain some finite piece of the reduction of $C[M]$; if the procedure stops because no more descendants of $L$ exist, or will be activated, then we gain an infinite reduction of $C[M]$.

In the following, we make this intuitive description more precise. We deviate from the practice up to now and allow for contexts with several holes. If $C$ is a context with $n$ holes, we write $C\left[L_{1}, \ldots, L_{n}\right]$ for the term obtained from $C$ by replacing the holes by $L_{1}, \ldots, L_{n}$ in that order. Moreover, we write $C[L, \ldots, L] \rightarrow C^{\prime}[L, \ldots, L]$ if the occurrences of $L$ displayed in $C^{\prime}[L, \ldots, L]$ are precisely the descendants of the occurrences of $L$ displayed in $C[L, \ldots, L]$.

Proposition 20. Let $L$ be a normal form.

1. Let

$$
C[L, \ldots, L] \rightarrow \cdots \rightarrow C^{\prime}\left[L, \ldots, L^{*} P[L, \ldots, L], \ldots, L\right]
$$

be a reduction until for the first time a descendant (displayed as $L^{*}$ ) of one of the $L$ 's shown in $C[L, \ldots, L]$ is activated. Then for every $M$,

$$
C[M, \ldots, M] \rightarrow \cdots \rightarrow C^{\prime}[M, \ldots, M P[M, \ldots, M], \ldots, M]
$$

is a reduction obtained by replacing every descendant of the $L$ 's by $M$.

2. Let $C[L, \ldots, L] \rightarrow \cdots$ be an infinite reduction in which no descendant of the displayed $L$ 's ever is activated. Then for every $M, C[M, \ldots, M] \rightarrow \cdots$ is an infinite reduction obtained by replacing every descendant of the $L$ 's by $M$.

Proof. Routine.

Theorem 21. The final collapse of $\mathfrak{A}_{\mathrm{SN}}$ is extensional.

Proof. Let $L, M$ be normal forms such that

$$
\text { (‡) } \forall C \in \mathcal{C}_{\mathrm{SN}} \forall N \in \mathrm{SN}^{0}(C[L N] \in \mathrm{SN} \leftrightarrow C[M N] \in \mathrm{SN}) .
$$

We shall prove that $C[L] \in \mathrm{SN}$ iff $C[M] \in \mathrm{SN}$ for all $C \in \mathcal{C}_{\mathrm{SN}}$. Suppose this is not the case, say $C[L] \notin \mathrm{SN}$ and $C[M] \in \mathrm{SN}$ for some $C \in \mathcal{C}_{\mathrm{SN}}$. We shall derive a contradiction by constructing an infinite reduction of $C[M]$ as follows: Let $\mathcal{R}: C[L] \rightarrow \cdots$ be an infinite reduction. If no descendant of $L$ ever is activated, then $\mathcal{R}^{\prime}: C[M] \rightarrow \cdots$ obtained by replacing every descendant of $L$ by $M$ is an infinite reduction by Proposition 20.2. Otherwise we consider the initial part of $\mathcal{R}$ up to the first moment in which some descendant of $L$ is activated:

$$
C[L] \rightarrow \cdots \rightarrow C^{*}\left[L, \ldots, L^{*} P[L, \ldots, L], \ldots, L\right] .
$$

This is the $A_{0} B_{1}$-edge in the diagram below. Now replace the activated descendant of $L$ by $M$. Observe that this term stays infinite (i.e. is not strongly normalizing). For, either

(i) $C^{*}[L, \ldots, \square, \ldots, L] \in \mathcal{C}_{\mathrm{SN}}$ and $P[L, \ldots, L] \in \mathrm{SN}$ : then we can apply $(\ddagger)$, or 
(ii) $C^{*}[L, \ldots, \square, \ldots, L] \notin \mathcal{C}_{\mathrm{SN}}$ : then $C^{*}[L, \ldots, \square, \ldots, L]$ contains a subterm that is not strongly normalizing and hence $C^{*}[L, \ldots, M P[L, \ldots, L], \ldots, L] \notin \mathrm{SN}$, or

(iii) $P[L, \ldots, L] \notin \mathrm{SN}$ : then also $C^{*}[L, \ldots, M P[L, \ldots, L], \ldots, L] \notin \mathrm{SN}$.

In case of a final $S$-redex contraction, there may be another activated descendant of $L$. That is,

$$
C^{*}[L, \ldots, M P[L, \ldots, L], \ldots, L] \equiv C^{*}\left[L, \ldots, L^{*} Q[L, \ldots, L], \ldots, L\right] .
$$

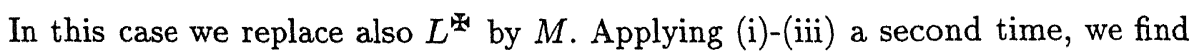
that this new term stays also infinite. By Proposition 20.1, we have a reduction

$$
C[M] \rightarrow \cdots \rightarrow C^{*}[M, \ldots, M P[M, \ldots, M], \ldots, M]
$$

which we depict by the $D_{0} D_{1}$-edge in the diagram. Observe that

$$
C^{*}[M, \ldots, M P[M, \ldots, M], \ldots, M] \equiv C^{*}[M, \ldots, M Q[M, \ldots, M], \ldots, M] .
$$

We now reiterate this procedure, using instead of $\mathcal{R}$ an infinite reduction

$$
\mathcal{R}^{*}: C^{*}[L, \ldots, M P[L, \ldots, L], \ldots, L] \rightarrow \cdots
$$

or

$$
\mathcal{R}^{*}: C^{W}[L, \ldots, M Q[L, \ldots, L], \ldots, L] \rightarrow \cdots
$$

which corresponds to the horizontal edge starting in point $A_{1}$. Note that $L^{*}$ and

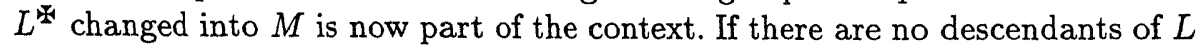
left, then

$C^{*}[L, \ldots, M P[L, \ldots, L], \ldots, L] \equiv C^{*}[M P] \equiv C^{*}[M, \ldots, M P[M, \ldots, M], \ldots, M]$

and we are done:

$$
C[M] \rightarrow \cdots \rightarrow C^{*}[M P] \rightarrow \cdots
$$

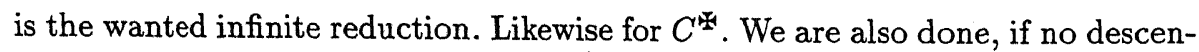
dant of $L$ ever is activated in $\mathcal{R}^{*}$ or $\mathcal{R}^{*}$. For, in that case we obtain an infinite reduction

$$
C[M] \rightarrow \cdots \rightarrow C^{*}[M, \ldots, M P[M, \ldots, M], \ldots, M] \rightarrow \cdots
$$

by Proposition 19.2; likewise for $C^{4}$. In the remaining case, we consider the initial part of $\mathcal{R}^{*}\left(\mathcal{R}^{*}\right)$ up to the first moment in which a descendant of the remaining descendants of $L$ is activated. This is the $A_{1} B_{2}$-edge in the diagram. Employing Proposition 19.1, we gain the edge $D_{1} D_{2}$. In this way, we proceed ad infinitum. 


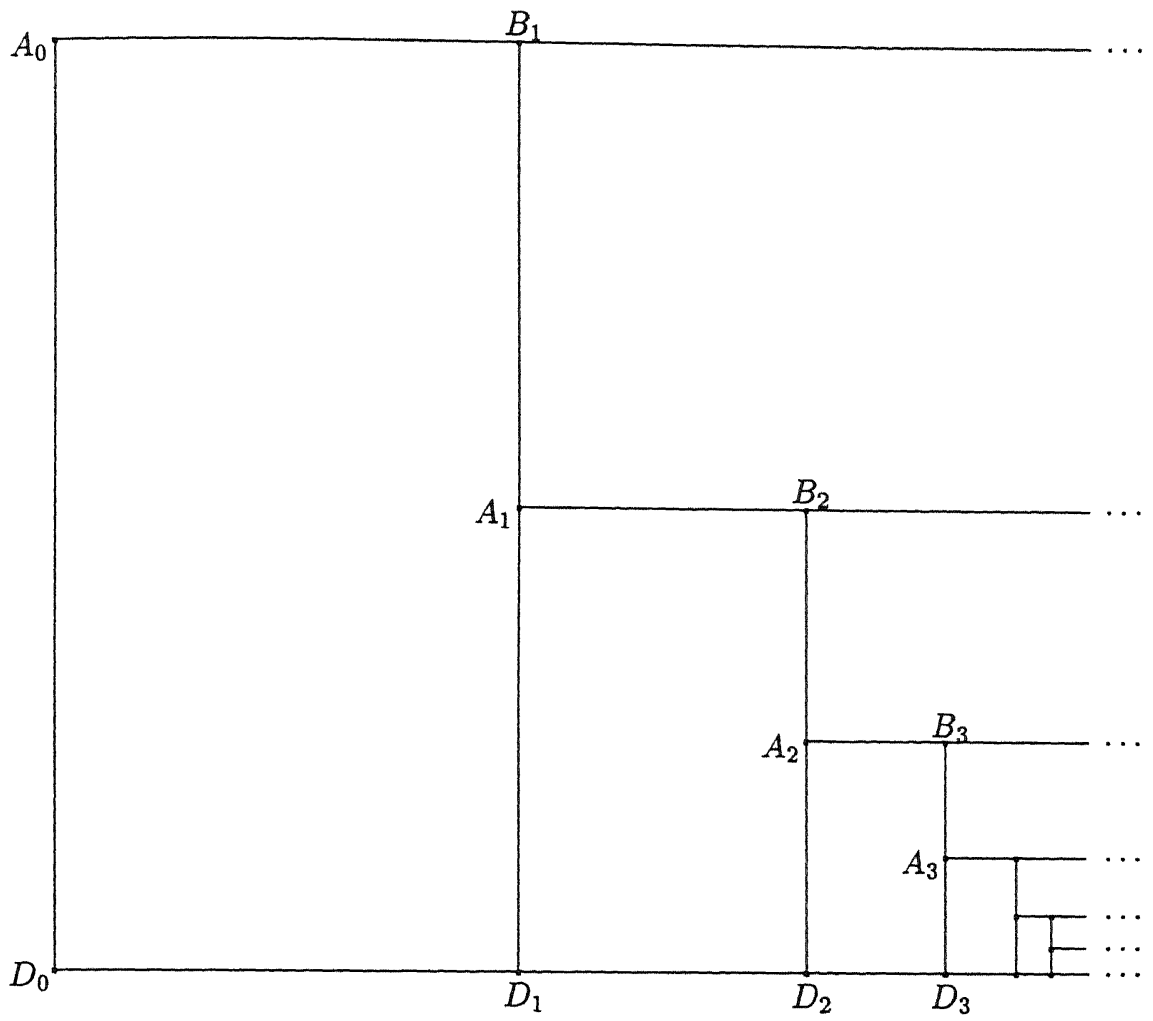

Remark. Let $\mathfrak{A}=\langle A, s, k, \cdot\rangle$ be a pca. We call $\operatorname{ker}(\mathfrak{A})$, the kernel of $\mathfrak{A}$, the subset of $A$ containing all elements generated by $k$ and $s$. So $\operatorname{ker}(\mathfrak{A})$ is defined by:

1. $k, s \in \operatorname{ker}(\mathfrak{A})$, and

2. if $a, a^{\prime} \in \operatorname{ker}(\mathfrak{A})$ and $a a^{\prime} \downarrow$, then $a a^{\prime} \in \operatorname{ker}(\mathfrak{A})$.

In case $\operatorname{ker}(\mathfrak{A})=A$, we call $\mathfrak{A}$ a minimal pca. Note that the nca $\mathfrak{A}_{\mathrm{SN}} / E_{\mathrm{fin}}$ is in fact minimal.

As observed in [Bet87], extensional nca's cannot be completed to a ca by adding some elements and completing the application operation. For, suppose $\mathfrak{A}=\langle A, s, k, \cdot\rangle$ is an extensional nca and $\mathfrak{A}^{\prime}$ is some completion of $\mathfrak{A}$. Choose $a, a^{\prime} \in A$ such that $a a^{\prime} \uparrow$ and put $\perp \equiv s(k a)\left(k a^{\prime}\right)$. Observe that $\perp a^{\prime \prime} \uparrow$ for every $a^{\prime \prime} \in A$, and hence $s(k(k k)) \perp a^{\prime \prime} \uparrow$ and $s(k(k s)) \perp a^{\prime \prime} \uparrow$ for every $a^{\prime \prime} \in A$. By 
extensionality, we have therefore $s(k(k s)) \perp=s(k(k k)) \perp$. But then

$$
s=s(k(k s)) \perp \cdot^{\prime} k=s(k(k k)) \perp \cdot^{\prime} k=k .
$$

By the preceding result, $\mathfrak{A}_{\mathrm{SN}} / E_{\mathrm{fin}}$ cannot be completed. So $\mathfrak{A}_{\mathrm{SN}} / E_{\mathrm{fin}}$ is both incompletable and minimal. This is an extra as compared to the construction of similar counterexamples to completability as in [Bet87] and [Klo82].

Question 22. It is an intriguing question to determine what the 'structure' of $\mathfrak{A}_{\mathrm{SN}} / E_{\mathrm{fin}}$ is, or how to find a suitable representation of its elements.

\section{References}

[AC95] A. Asperti and A. Ciabattoni. Effective applicative structures. In Proceedings of the 6th biennial conference on Category Theory in Computer Science (CTCS'95), volume 953 of Lecture Notes in Computer Science, pages 81-95, 1995.

[Bar84] H.P. Barendregt. The Lambda Calculus, its Syntax and Semantics, volume 103 of Studies in Logic and the Foundations of Mathematics. North-Holland Publishing Company, revised edition, 1984. (Second printing 1985).

[BB79] J. Baeten and B. Boerboom. $\Omega$ can be anything it should not be. Indagationes Mathematicae, 41:111-120, 1979.

[Bee85] M.J. Beeson. Foundations of Constructive Mathematics, volume 6 of Ergebnisse der Mathematik und ihrer Grenzgebiete, 3.Folge. Springer-Verlag, 1985.

[Bet87] I. Bethke. On the existence of extensional partial combinatory algebras. Journal of Symbolic Logic, 52(3):819-833, 1987.

[Bet91] I. Bethke. Finite type structures within combinatory algebras. Annals of Pure and Applied Logic, 55:101-123, 1991.

[BI93] A. Berarducci and B. Intrigila. Some new results on easy lambda-terms. Theoretical Computer Science, 121:71-88, 1993.

[Bur82] P. Burmeister. Partial algebras - survey of a unifying approach towards a two-valued model theory for partial algebras. Algebra Universalis, 15:306$358,1982$.

[DJ90] N. Dershowitz and J.-P. Jouannaud. Rewrite systems. In J. van Leeuwen, editor, Formal Methods and Semantics, Handbook of Theoretical Computer Science, Volume B, chapter 6, pages 243-320. MIT Press, 1990.

[Eng81] E. Engeler. Algebras and combinators. Algebra Universalis, 13:389-392, 1981.

[Grä79] G. Grätzer. Universal algebra. Second edition. Springer-Verlag, 1979.

[HS86] J.R. Hindley and J.P. Seldin. Introduction to Combinators and $\lambda$-calculus, volume 1 of London Mathematical Society Student Texts. Cambridge University Press, 1986.

[Jac75] G. Jacopini. A condition for identifying two elements of whatever model of combinatory logic. In C. Böhm, editor, $\lambda$-Calculus and Computer Science Theory, volume 37 of Lecture Notes in Computer Science, pages 213-219. SpringerVerlag, 1975.

[JZ85] G. Jacopini and M. Venturini Zilli. Easy terms in the lambda-calculus. Fundamenta Informaticae, VIII(2):225-233, 1985.

[Klo82] J.W. Klop. Extending partial combinatory algebras. Bulletin of the European Association for Theoretical Computer Science, 16:472-482, 1982. 
[Klo92] J.W. Klop. Term rewriting systems. In D. Gabbay S. Abramsky and T. Maibaum, editors, Handbook of Logic in Computer Science, Volume II. Oxford University Press, 1992.

[Mei95] K. Meinke. A survey of higher-order algebra. Technical Report U.U.D.M. 1995:39, ISSN 1101-3591, University of Uppsala, 1995.

[Mit9?] J.C. Mitchell. Introduction to Programming Language Theory. Forthcoming.

[Plo72] G.D. Plotkin. A set-theoretical definition of application. Technical Report Memorandum MIP-R-95, School of Artificial Intelligence, University of Edinburgh, 1972.

[Sco76] D.S. Scott. Data types as lattices. SIAM J. Comput., 5:522-587, 1976.

[Str91] T. Streicher. Semantics of type theory : correctness, completeness and independence results. Birkhäuser Verlag, 1991.

[Str68] H.R. Strong. Algebraically generalized recursive function theory. IBM J. Research and Development, 12:465-475, 1968.

[Swan79] Open problems; Swansea $\lambda$-calculus meeting; 21 September 1979. Bulletin of the European Association for Theoretical Computer Science, 10:136-140, 1980.

[TvD88] A.S. Troelstra and D. van Dalen. Constructivism in Mathematics. An Introduction. Volume II, volume 123 of Studies in Logic and the Foundations of Mathematics. North-Holland Publishing Company, 1988.

[Wag69] E. Wagner. Uniformly reflexive structures. Trans. American Math. Society, 144:1-41, 1969. 